\title{
The role of homocysteine in seminal vesicles remodeling in rat
}

\author{
Adel Ghoul ${ }^{1}$, Elara Moudilou ${ }^{2}$, Mohamed El Hadi Cherifi ${ }^{3}$, Fouzia Zerrouk ${ }^{1}$, \\ Billel Chaouad ${ }^{1}$, Anissa Moulahoum ${ }^{1}$, Souhila Aouichat-Bouguerra ${ }^{4}$, \\ Khira Othmani' ${ }^{1}$ Jean-Marie Exbrayat ${ }^{2}$, Yasmina Benazzoug ${ }^{1}$
}

${ }^{1}$ Laboratory of Cellular and Molecular Biology (BCM), Extracellular Matrix, Faculty of Biological Sciences (FSB), USTHB, El Alia, Algiers, Algeria

${ }^{2}$ University of Lyon, UMRS 449, General Biology, Reproduction and Comparative Development, Catholic University of Lyon, EPHE/PSL, Lyon Cedex 02, France

${ }^{3}$ EPH Bologhine Ibn Ziri, Central Laboratory of Biology, Algiers, Algeria

${ }^{4}$ Laboratory of Cellular and Molecular Physiopathology, Faculty of Biological Sciences, USTHB, Algiers, Algeria

\begin{abstract}
Introduction. Elevated plasma homocysteine (Hcy) levels have been associated with several tissue injuries including heart and liver fibrosis. In these diseases, hyperhomocysteinemia (Hhcy) plays a major role in modulating the alteration of the balance between matrix metalloproteinases (MMP) and tissue inhibitors of metalloproteinases (TIMPs), leading to the pathological accumulation of extracellular matrix (ECM) proteins. Since the effect of Hhcy on ECM of seminal vesicle was not studied, the aim of our research was to check if Hcy can induce a remodeling within seminal vesicles ECM.

Material and methods. The study was conducted in 22 adult male Wistar rats. The rats were divided into two groups: a control group, which received standard diet and tap water; the treated group received the same diet and water supplemented with solution of L-methionine $(200 \mathrm{mg} / \mathrm{kg}$ b.w./day) for 6 months. Plasma homocysteine concentration was measured. Histological changes were observed with light microscope. The presence of collagen I and III and metalloproteinases (2, 3, 7 and 9) in the seminal vesicles was examined using immunohistochemistry and Western blotting.

Results. Plasma Hcy levels increased significantly after methionine treatment and interfered significantly with body weight in treated rats. The content of fibrillar collagens (I and III) in the wall of seminal vesicles was elevated in hyperhomocysteinemic rats. Moreover, we found that hyperhomocysteinemia increased the expression of MMP-2, -3, -7 and -9 in seminal vesicles of experimental rats.

Conclusions. Increased plasma concentration of Hcy accompanied by the accumulation of collagen and upregulation of MMPs in rat seminal vesicles might contribute to the remodeling of seminal vesicles. Folia Histochemica et Cytobiologica 2017, Vol. 55, No. 2, 62-73)
\end{abstract}

Key words: hyperhomocysteinemia; rat; seminal vesicles; metalloproteinases; collagen I; collagen III; IHC; Western blot

Correspondence address: A. Ghoul, Ph.D.

Université des Sciences et de la Technologie

Houari-Boumédiène, Alger

BP 32 El Alia 16111, Bab Ezzouar 16111, Algérie

tel.: +21321247913

e-mail: ghoul.adel@yahoo.fr

\section{Introduction}

Homocysteine (Hcy) is a sulfur containing amino acid formed during the metabolism of methionine, an essential amino acid derived from the diet [1]. The hyperhomocysteinemia (Hhcy) is characterized by 
a high plasma Hcy concentration, which is associated with several pathological conditions like cardiovascular diseases [2], cerebral [3] and renal failure [4]. The pathological processes by which homocysteine promotes organ damage have yet not been fully elucidated. Oxidative stress [5] and activation of pro-inflammatory factors [6] have been proposed to explain the pathogenic effects of Hhcy. Previous studies have revealed that these processes induced by Hhcy were associated with initiation and progression of fibrosis characterized by an accumulation of extracellular matrix (ECM) proteins, particularly collagen and an imbalance of MMPs/TIMPs system, which also plays a determinant role in the regulation of collagen tissue turnover [7, 8].

Matrix metalloproteinases (MMPs) are a family of zinc-dependent proteolytic enzymes, classified into five groups: gelatinases (MMP-2 and MMP-9), mainly targeting denatured interstitial collagen (gelatin) and type I and IV collagen fibers; stromelysins (MMP-3, MMP-10, and MMP-11), targeting non-collagen molecules and proteoglycans; collagenases (MMP-1, MMP-8, MMP-13, and MMP-18), targeting fibrillar forms of collagen; matrilysins (MMP-7 and MMP-26) and MT-MMPs (MMP-14, MMP-15, MMP-16, MMP-17, MMP-24, and MMP-25), a group of transmembrane enzymes not only cleaving ECM components, but also activating other MMPs [9-12]. MMPs are implicated in remodeling of many connective tissues in physiological and pathological processes such as reproduction, embryonic development, morphogenesis, angiogenesis and fibrosis of such organs as liver, kidney and pulmonary [13-18].

Up to date, many studies have been performed on the expression and function of MMPs and tissue inhibitors of metalloproteinases (TIMPs) in many female reproductive processes, including menstruation, atresia of ovarian follicles, embryo implantation and during involutive processes of the uterus [19-21]. In male, MMPs have a role in the fertilization process that requires breakdown of the physiological barrier during sperm penetration through the zona pelluci$d a$ and egg's plasma membrane [22]. Furthermore, MMPs and their tissue inhibitors have a role in the maintenance of internal environment homeostasis inside seminiferous tubules and are therefore involved in spermatogenesis [23]. In this context, relationships between MMPs and seminal parameters have been reported [24]. Additionally to these physiological effects of MMPs, it has been demonstrated that MMPs are involved in seasonal cycle of reproduction of Libyan jird, in which they are highly expressed in the seminal vesicles during the breeding period to promote ECM remodeling of seminal vesicles, related to the reproduction activity of this rodent in seasonal period [25].

Thus, considering the importance of MMPs in male reproduction and also the unclear aspect about pathological remodeling of seminal vesicles, the aim of our study was to investigate the effects of Hhcy on the expression of MMPs and fibrillary collagens (type I and III) in seminal vesicles of Wistar rats.

\section{Material and methods}

Animals. Twenty-two adult male Wistar rats were purchased from Pasteur Institute of Algiers, Algeria. The animals were housed in plastic cages and allowed to acclimatize under standard conditions $\left(25^{\circ} \mathrm{C}\right.$ with 12 -hour light/dark cycles). They were given free access to tap water and standard diet: instead of standard rat chow. Animals were randomly divided into two groups as follow: the control group ( $\mathrm{n}=10$, mean mass $245 \mathrm{~g}$ at the start of experiment) was fed a standard laboratory diet, the experimental group $(n=12$, mean mass $252 \mathrm{~g}$ ) received a standard diet and water supplemented with L-methionine (Sigma-Aldrich, France; $200 \mathrm{mg} / \mathrm{kg}$ b.w./day) for 6 months. This treatment resulted in increased plasma homocysteine concentration $(\sim 30 \mu \mathrm{M}$ in the experimental group $v s . \sim 20 \mu \mathrm{M}$ in the control group), as shown in our previous study [26]. All experiments complied with the Algerian legislation (Law Number 12-235/2012) inherent to protection of animals designed to experimental and other scientific purposes as well with the guidelines of the Algerian Association of Experimental Animal Sciences (AASEA) and were specifically approved by the latter (AASEA authorization number 45/DGLPAG/DVA/SDA/14).

Body and seminal vesicle's mass. At the end of the experimental period, the animals were sacrificed and seminal vesicles were immediately excised. For all animals, body and seminal vesicles mass was recorded at the time of sacrifice. Then, parts of the seminal vesicles (left and right) were fixed in Bouin-Hollande's fluid for histological and histochemical studies and in $10 \%$ formalin for immunohistochemical analysis.

\section{Measurements of plasma homocysteine concentration.} Whole blood samples from rats were collected into tubes containing ethylene diaminetetra-acetic acid (K3, EDTA, FL medical, Torreglia, Italy). To obtain plasma samples, whole blood samples were immediately centrifuged at $1000 \mathrm{~g}$ for $10 \mathrm{~min}$ at $4^{\circ} \mathrm{C}$. Total Hcy concentrations were determined on the Abbott automated immunoassay analyzer (AxSYM system, Abbott, Abbott Park, IL, USA). The determination of Hcy concentration was based on fluorescence polarization immunoassay technology FPIA [27]. All forms of homocysteine (mixed disulfide and bound homocysteine) in sample 
are reduced to free homocysteine by dithiothreitol, which is then converted to S-adenosyl-L-homocysteine (SAH) by SAH hydrolase and excess adenosine. Mouse monoclonal antibody against SAH is used along with labeled fluorescein tracer. The SAH and labeled Fluorescein Tracer in the sample compete for the sites on the monoclonal antibody molecule. The intensity of polarized fluorescence light was measured by the FPIA optical assembly.

Histological and histochemical techniques. Seminal vesicles were fixed for $48 \mathrm{~h}$ in Bouin-Hollande's solution. Then seminal vesicles were dehydrated in an ascending series of ethanol, cleared in two changes of xylene and embedded in liquid paraffin at $58^{\circ} \mathrm{C}$. Sections of $5 \mu \mathrm{m}$ thickness were cut using rotary microtome (Leica RM 2125RT, Wetzlar, Germany) and mounted on clean slides (Superfrost plus, Menzel Gläser, Thermo scientific, Braunschweig, Germany).

To assess the morphology of seminal vesicles sections were stained with picrosirius red (PSR) for the detection of collagen fibers [28]. Glycosaminoglycans (GAGs) were detected using $1 \%$ alcian blue (AB) 8GX (Sigma-Aldrich, St. Louis, MO, USA) dissolved in $3 \%$ acetic acid, $\mathrm{pH} 2.5$ for $20 \mathrm{~min}$ to stain negatively charged glycoproteins as well as GAG chains of proteoglycans [29]. Carbohydrates were detected using periodic acid Schiff reaction (PAS); for that, the sections were oxidized in $1 \%$ periodic acid solution for $10 \mathrm{~min}$ at room temperature; after washing in distilled water, the sections were immersed in Schiff's reagent for $10 \mathrm{~min}$. All carbohydrate molecules (both neutral and acidic) became red-stained [30].

Immunohistochemical staining. After fixation of seminal vesicles in $10 \%$ formalin for $24 \mathrm{~h}$ and embedding in paraffin, sections $(5 \mu \mathrm{m})$ were subjected to immunohistochemical staining using the Vectastain ABC kit: instead of avidin biotin peroxidase complex for detection of collagens (type I and III) and MMPs (2, 3, 7 and 9). The sections were immunolabeled with antibodies against type I collagen (ab34710, Abcam, Cambridge, UK), type III collagen (ab7778, Abcam), MMP-2 (MAB13405, Millipore, California, USA), MMP-3 (MAB3369, Millipore), MMP-7 (MAB3315, Millipore) and MMP-9 (MAB3309, Millipore) and the reaction was amplified using the Vectastain kit (UNIVERSAL VECATSTAIN Kit ABC, Vector, Burlingame, California, USA) method. All antibodies were diluted 1:100 in phosphate-buffered saline (PBS). The serial consecutive $5-\mu \mathrm{m}$ sections were dewaxed in cyclohexane $(2 \times 5 \mathrm{~min})$, rehydrated in $100 \%, 96 \%$ and $70 \%$ ethanol for $5 \mathrm{~min}$ each, and then the slides were rinsed with tap water $(2 \times 5 \mathrm{~min})$. For the retrieval of collagen I and III epitopes, the slides were heated in water-bath for $20 \mathrm{~min}$ at $95^{\circ} \mathrm{C}$ using a Vector antigen unmasking solution (H3301, Vector) and for the retrieval of MMP-2, -3, -7 and MMP-9 epitopes, the sections were placed in antigen unmasking solution, citrate-based (H-3300, Vector), using a pressure cooker heating for $1 \mathrm{~min}$ and then all slides were cooled to room temperature for $30 \mathrm{~min}$. The endogenous peroxidase activity was blocked with $3 \%$ hydrogen peroxide in PBS $1 \mathrm{X}$ for $20 \mathrm{~min}$ followed by rinsing with PBS for $3 \mathrm{~min}$, and after that, slides were incubated with a $10 \%$ normal horse serum in PBS for $1 \mathrm{~h}$ at room temperature (RT) to block non-specific binding sites. The slides were incubated with primary antibodies (against type I and type III collagens) for $1 \mathrm{~h}$ at RT or overnight at $4^{\circ} \mathrm{C}$ (MMP-2, -3, -7 and MMP-9), and after that slides were washed in PBS $(2 \times 5 \mathrm{~min})$. Next, the slides were incubated with prediluted secondary antibody, anti-mouse IgG (VECATSTAIN Kit, Vector) for $30 \mathrm{~min}$, followed by two baths in PBS (5 min) and exposed to streptavidin complex for $30 \mathrm{~min}$. Staining was also visualized using a Vector NovaRED Peroxidase (HRP) Substrate Kit (Vector). The sections were counterstained with hematoxylin (hematoxylin QS, H-3404; Vector), dehydrated, mounted, and then observed with a Nikon Eclipse E400 light microscope (Nikon, Tokyo, Japan), and digitized images were obtained using a Nikon DXM 1200 digital camera. The negative controls for secondary antibody were performed by treating slides according to the protocol, but omitting the primary antibody.

Western blot analysis. Seminal vesicles were dissected, immediately frozen in liquid nitrogen and stored at $-80^{\circ} \mathrm{C}$ before protein extraction. The tissues were homogenized in RIPA buffer containing $\mathrm{NaCl} 150 \mathrm{mM}$, $1 \%$ Nonidet NP-40, $0.1 \%$ sodium dodecyl sulfate (SDS), $0.5 \%$ sodium deoxycholate, $50 \mathrm{mM}$ Tris-HCL at $\mathrm{pH}$ 8.0 and protease inhibitor cocktail (Mini EASYpack, Roche Diagnostics GmbH, Mannheim, Germany) at $\mathrm{pH}$ 7.8. After three freeze/thaw cycles and incubation on ice for $10 \mathrm{~min}$, the homogenate was centrifuged at $15,000 \mathrm{~g}$ at $4^{\circ} \mathrm{C}$ for $30 \mathrm{~min}$, the supernatants collected and total protein concentration determined with the bicinchoninic acid assay (BCA, Sigma-Aldrich). The total proteins $(20 \mu \mathrm{g})$ were mixed with loading buffer (final concentrations: $0.062 \mathrm{M}$ Tris $\mathrm{HCl}, \mathrm{pH}$ 6.8, $1 \%$ SDS, $0.02 \%$ bromophenol blue, $10 \%$ glycerol) under reducing and denaturing conditions and subjected to SDS-PAGE using $12 \%$ polyacryamid gels. The separated proteins were transferred to a PVDF membrane (ISEQ00010 Immobilon-PSQ Membrane, PVDF, $0.2 \mu \mathrm{m}$, Merck Millipore, Billerica, MA, USA), followed by blocking in 5\% non-fat dry milk in Tris-buffered saline containing $0.1 \%$ Tween-20 (TBST) for $1 \mathrm{~h}$ at RT. Thereafter, the membranes were incubated with primary antibodies against MMP-2 (1:500), MMP-3 (1:1000), MMP-7 (1:4000), MMP-9 (1:2000). The blots were rinsed three times in TBST, $5 \mathrm{~min}$ each. The membranes were then incubated for $1 \mathrm{~h}$ with anti-mouse-horseradish peroxidase $[\operatorname{IgG}(\mathrm{H}+\mathrm{L})$, Vector, Burlingame, CA, USA] for $1 \mathrm{~h}$ at final dilution 1:10 000 . After washing, the immune complex was detected with an 


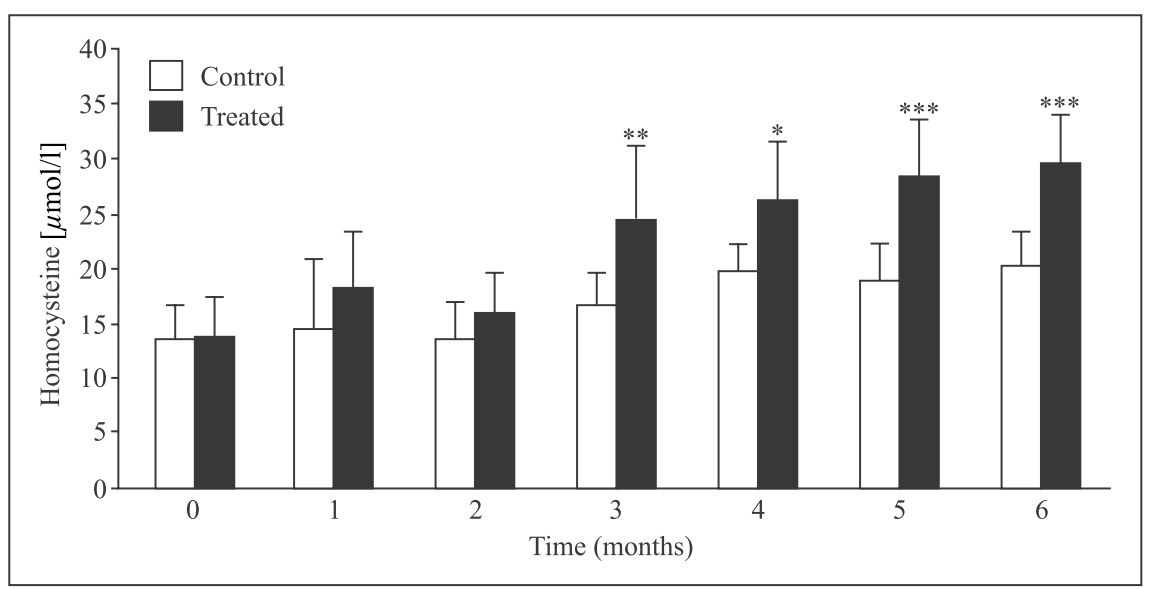

Figure 1. Plasma homocysteine concentrations in the control and methionine-treated rats. Results are expressed as mean \pm standard error. $*, * *, * *$ Statistically significant differences at the same time between the control and methionine-treated rats, $\mathrm{p}<0.05, \mathrm{p}<0.01$, and $\mathrm{p}<0.001$, respectively.

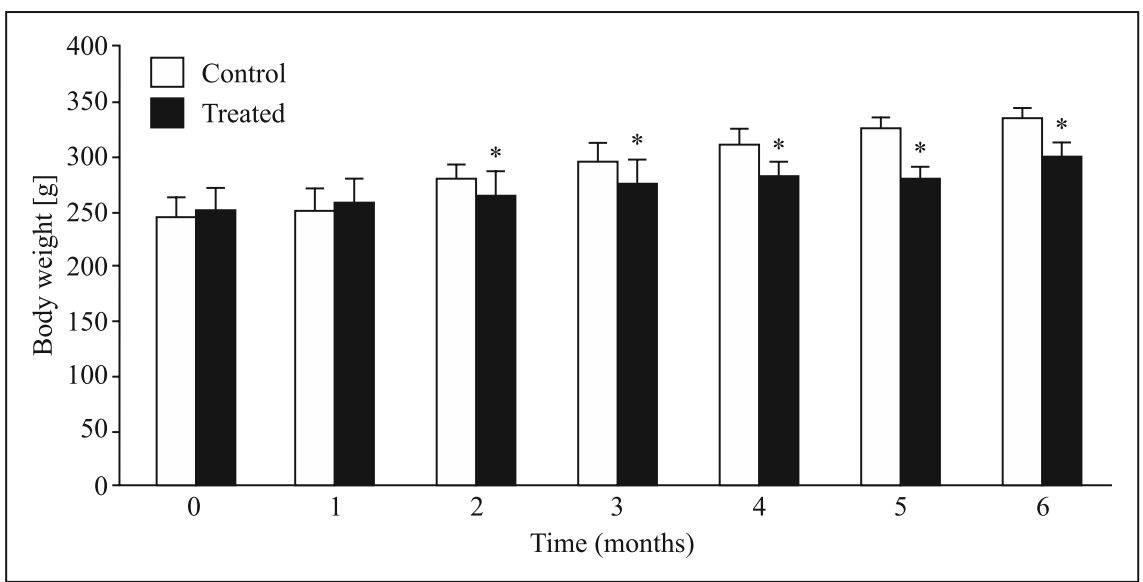

Figure 2. Effect of hyperhomocysteinemia on body mass of Wistar rats. Results are expressed in grams as mean \pm standard error. *Statistically significant difference between the control and methionine-treated rats, $\mathrm{p}<0.05$.

enhanced chemiluminescence detection system (GE Healthcare, Amersham ECL, Buckinghamshire, UK).

Statistical analysis. Statistical analysis was performed using STATISTICA software (version 6; StatSoft Inc, Maisons-Alfort, Paris, France). Results are presented as mean \pm standard error of mean. Mann-Whitney U, non-parametric test was used to make comparison between groups (control $v s$. treated rats). $\mathrm{P}<0.05$ was considered statistically significant.

\section{Results}

\section{Plasma homocysteine levels}

We found that 6 months on an L-methionine diet resulted in a significant $(\mathrm{p}<0.001)$ increase in plasma Hcy levels in treated rats as compared with control rats. Thus, the levels of plasma Hcy increased by $117 \%$ in the methionine-treated group $(13.96 \pm 3.43$ vs. $29.90 \pm 4.14 \mu \mathrm{mol} / \mathrm{L}$ ), and by $49 \%$ in the control group $(13.69 \pm 3.17 v s .20 .43 \pm 2.97 \mu \mathrm{mol} / \mathrm{L})$ over 6 months of the experimental period (Fig. 1).

\section{Changes of body and seminal vesicles mass}

Body weight gain (Fig. 2) was significantly smaller in the treated than in the control rats, from the second month until the end of experimental period $(\mathrm{P}<0.05)$. Thus, the body weight gain was approximatively $27 \%$ in the control rats and $19 \%$ in treated rats at the end of experimental period.

However, the mass of seminal vesicles in the methionine-treated rats did not differ significantly from that of the control rats $(5.06 \pm 0.53 \mathrm{mg} / \mathrm{g} v s .4 .59 \pm 0.77 \mathrm{mg} / \mathrm{g})$. 

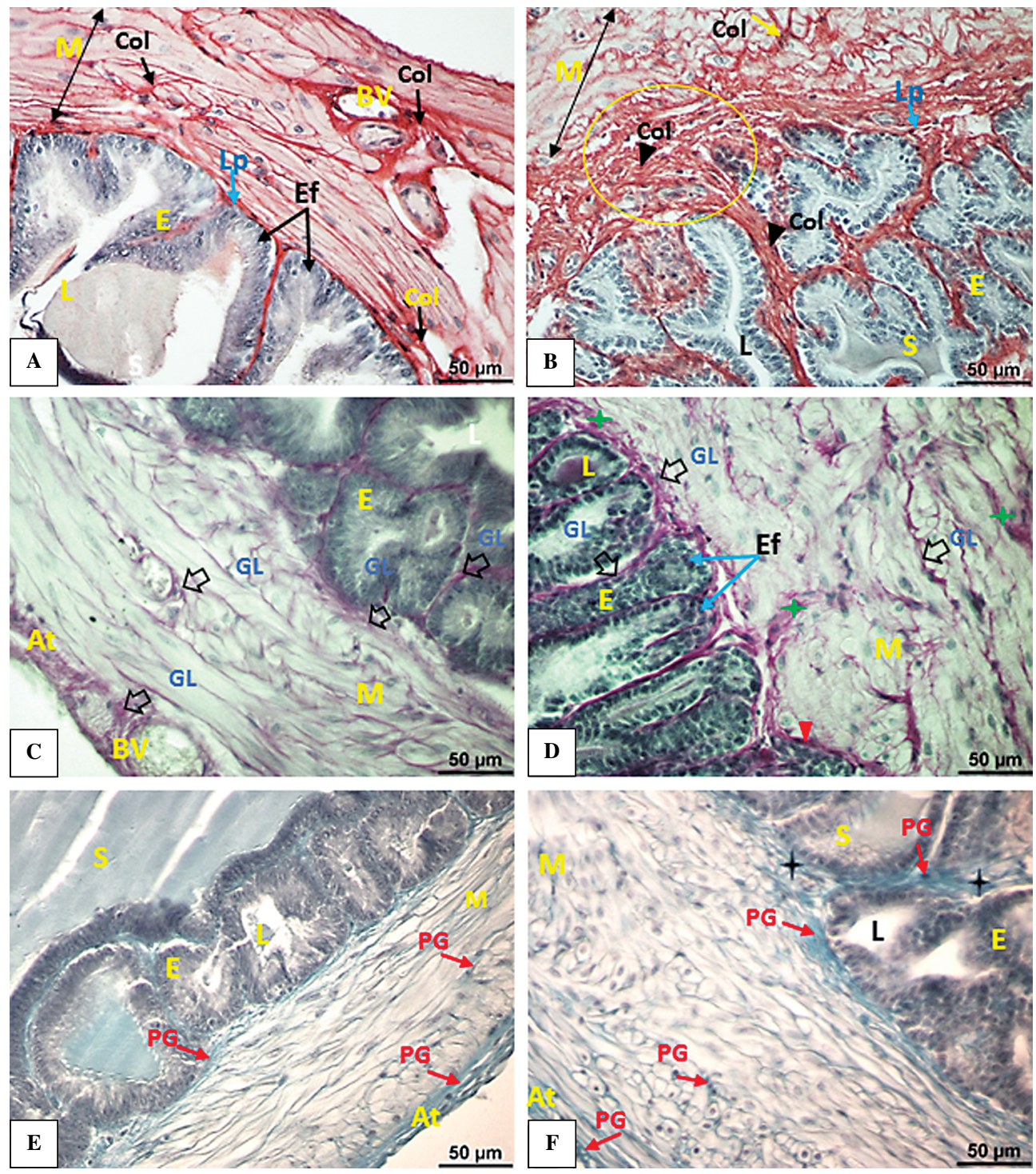

Figure 3. Representative microphotographs of seminal vesicles of the control (A, C, E) and methionine-treated rats (B, D, F). A. Picrosirius red staining reveals thin red collagen fibers (black arrow); B. Increased collagen staining in Lp (arrowhead, yellow circle) and in connective tissue surrounding SMC (black arrow); C. PAS staining reveals delicate pink color of glycoproteins (black arrow); D. Increased reactivity of PAS reaction (black arrow, green star); E. Moderate intensity of alcian blue staining of proteoglycans (red arrow); F. Small increase of proteoglycans presence in Lp (red arrow, black star). Abbreviations: At — adventitial tissue; BV — blood vessels; Col — collagen; E — epithelium; Ef — epithelial folds; GL — glycoproteins; L - lumen; Lp - lamina propria; M - muscularis externa; PG - proteoglycan; S - secretion; SMC - smooth muscle cells. Histological sections were stained as described in Material and methods, scale bars: $50 \mu \mathrm{m}$.

\section{Morphology of the seminal vesicles of methionine- -treated and control rats}

The seminal vesicles wall consists of three main layers: epithelium, lamina propria and muscular wall. The inner layer is composed of many epithelial folds, bordered with a pseudostratified epithelium (Fig. 3A), consisting of columnar and basal cells. It surrounds an irregular lumen containing the seminal secretion. The epithelium covers a rich vascularized lamina propria of loose connective tissue, connecting to the outer (muscular) wall. The connective tissue consists of strong collagenous fiber bundles, which stained with PSR (Fig. 3A, B), glycoproteins stained with PAS (Fig. 3C, D) and weak acidic proteoglycans stained with $\mathrm{AB}$ at $\mathrm{pH} 2.5$ (Fig. 3E, F).

In the methionine-treated rats, large and thick red collagen fibers were exhibited in the lamina propria and connective tissue surrounding the smooth muscle 


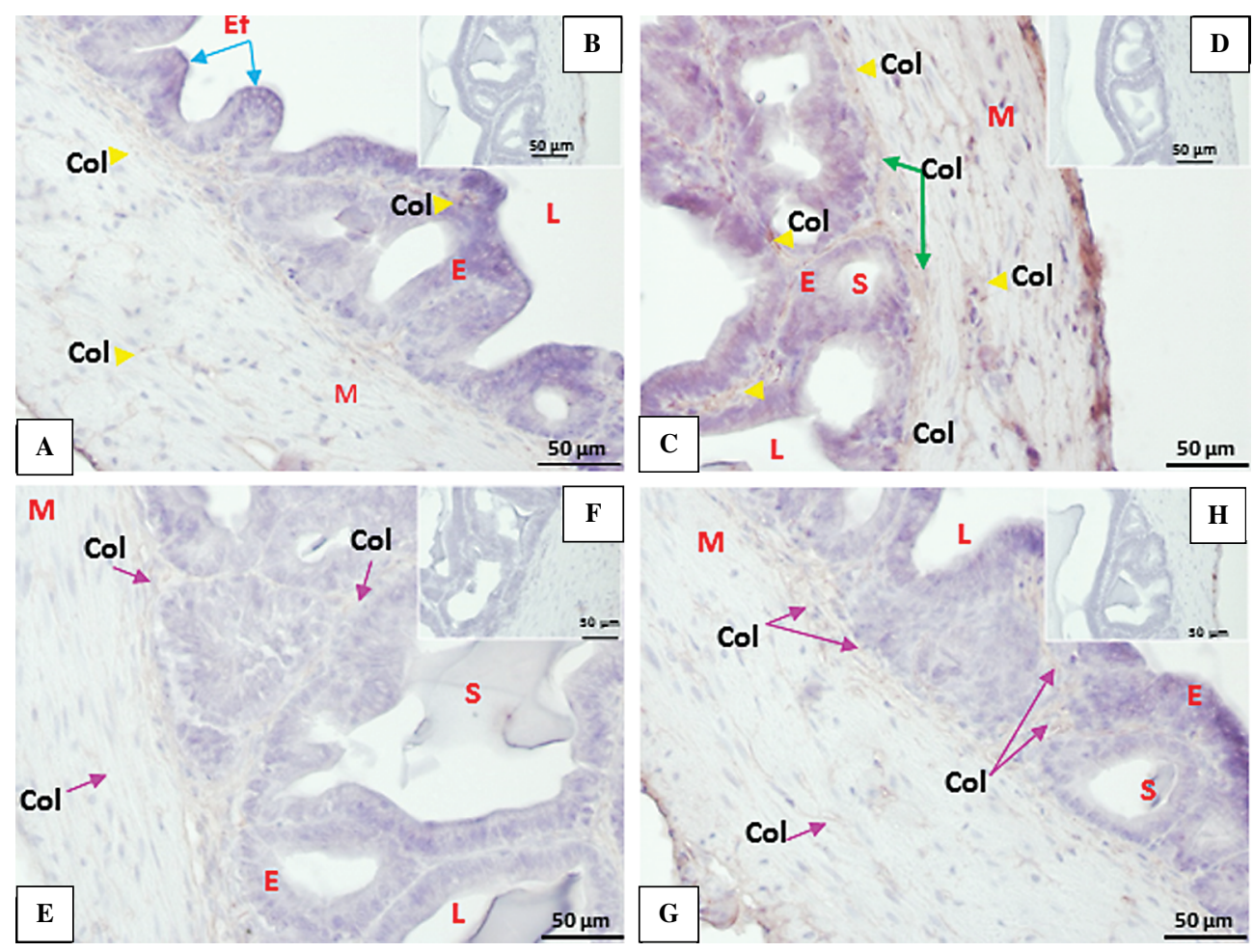

Figure 4. Immunohistochemical localization of collagen type I and type III in the seminal vesicles of the control (A, E) and methionine-treated rats $(\mathrm{C}, \mathrm{G})$. A. Weak immunoreactivity of type I collagen (arrowhead); C. pronounced immunoreactivity of collagen type I (yellow arrowhead, green arrow); E. Weak immunoreactivity of type III collagen (purple arrow); G. Small increase of collagen III immunoreactivity (arrow). Inserts: B, D, F, H - negative controls. Abbreviations as in the description of Figure 3. Histological sections were stained by immunohistochemical technique as described in Material and methods, scale bars: $50 \mu \mathrm{m}$.

layer (Fig. 3B), Inversely, in the control rats the collagen fibers were thinner and showed a less prevalent red staining (Fig. 3A) compared to treated rats.

Following staining with PAS method, staining differences of the vesicles' wall were observed between the treated and control rats (Fig. 3C, D). More staining of glycoproteins in connective tissue of lamina propria, around blood vessels and surrounding smooth muscle cells was found in the treated rats (Fig. 3D) compared to the control ones (Fig. 3C).

Methionine induced few changes of proteoglycans pattern. So, in lamina propria the intensity of $\mathrm{pH} 2.5$ alcian blue staining showed a small increase (Fig. 3F). Whereas, no change was observed in the connective tissue surrounding smooth muscle and adventitial fibers (Fig. 3E). In the control rats the presence of proteoglycans was detected in the lamina propria, the connective tissue surrounding smooth muscles and adventitial fibers.

\section{Changes of collagen I and III immunoreactivity in rat seminal vesicles}

Figure 4 (A, C, E and G) shows microphotographs of the seminal vesicles immunostained for type I and III collagen. Negative controls prepared without primary antibody are also shown in Figure 4 (B, D, F, H).

In the seminal vesicles of the control rats, collagen type I was detected in the ECM of lamina propria and the connective tissue surrounding the smooth muscle layer (Fig. 4A). In the treated rats, type I collagen immunostaining increased in both the superficial and deep layers of lamina propria, and connective tissue surrounding smooth muscles (Fig. 4C). In control rats, type III collagen (Fig. 4E) was weakly immunopositive in the ECM of lamina propria and connective tissue surrounding the smooth muscle. In the methionine-treated rats, type III collagen was slightly pronounced in the ECM of lamina propria (Fig. 4G).

In the negative controls, types I and III collagen were not detected in both treated and control rats.

Immunohistochemical expression of MMP-2, -3, -7 and -9 in seminal vesicles of control and methionine-treated rats

Collagen accumulation in seminal vesicles depends on both its synthesis, and its degradation by proteinases. Therefore, the immunoexpression patterns of 
Table 1. Summary of the immunoreactivities of MMP-2, -3, -7 and -9 in seminal vesicles of control and methionine-treated rats

\begin{tabular}{|c|c|c|c|}
\hline \multirow[t]{2}{*}{ Type of MMP } & \multirow[t]{2}{*}{ Cell type } & \multicolumn{2}{|c|}{ Intensity of immunoreactivity } \\
\hline & & Methionine-treated rats $(n=5)$ & Control rats $(n=5)$ \\
\hline \multirow[t]{9}{*}{ MMP-2 } & Epithelial cells & & \\
\hline & Nuclei & + & +++ \\
\hline & Cytoplasm & + & +++ \\
\hline & Smooth muscle cells & & \\
\hline & Nuclei & - & - \\
\hline & Cytoplasm & - & +++ \\
\hline & Fibroblast-like cells & & \\
\hline & Nuclei & - & - \\
\hline & Cytoplasm & - & + \\
\hline \multirow[t]{6}{*}{ MMP-9 } & Epithelial cells & & \\
\hline & Nuclei & - & ++ \\
\hline & Cytoplasm & - & ++ \\
\hline & Smooth muscle cells & & \\
\hline & Nuclei & - & - \\
\hline & Cytoplasm & + & +++ \\
\hline \multirow[t]{7}{*}{ MMP-3 } & Epithelial cells & & \\
\hline & Nuclei & +++ & + \\
\hline & Cytoplasm & +++ & + \\
\hline & Blood vessels & - & ++ \\
\hline & Smooth muscle cells & & \\
\hline & Nuclei & - & ++ \\
\hline & Cytoplasm & - & + \\
\hline \multirow[t]{6}{*}{ MMP-7 } & Epithelial cells & & \\
\hline & Nuclei & ++ & - \\
\hline & Cytoplasm & +++ & ++ \\
\hline & Smooth muscle cells & & \\
\hline & Nuclei & - & ++ \\
\hline & Cytoplasm & - & - \\
\hline
\end{tabular}

Sections were stained as described in Material and methods. The intensity of immunoreactivity was assessed as: negative $(-)$, weak $(+)$, moderate $(++)$, and strong $(+++)$.

MMP-2, MMP-3, MMP-7 and MMP-9 were studied in seminal vesicles (Table 1 and Fig. 5). In the control rats, a weak MMP-2 immunoreactivity (Ir) was localized in the cytoplasm of all the epithelial cells and in few nuclei of the same cells (Fig. 5A). In the methionine-treated rats increased MMP-3-Ir was found in epithelial cells (nuclear and cytoplasmic staining), blood vessels, fibroblast-like cells and cytoplasm of smooth muscle cells compared to the control rats (Fig. 5C).

In seminal vesicles of the control rats, a weak immunoreactivity of MMP-9 was seen only in the cytoplasm of smooth muscles (Fig. 5E), with higher intensity in the treated rats than in the control animals. Moreover, MMP-9 staining was detected both in a few nuclei and cytoplasm of epithelial cells (Fig. 5G). In the control rats, MMP-3 was detected in the cytoplasm, and few nuclei of epithelial cells, whereas smooth muscles did not show any immunostaining (Fig. 5I). In the methionine-treated rats, MMP-3 was immunoexpressed with some moderate degrees in smooth muscles, blood vessels and it was also weakly expressed in the cytoplasm and nuclei of few epithelial cells (Fig. 5K). In the control rats, the immunodetection of MMP-7 revealed a very strong immunoreactivity in the cytoplasm and in few nuclei 


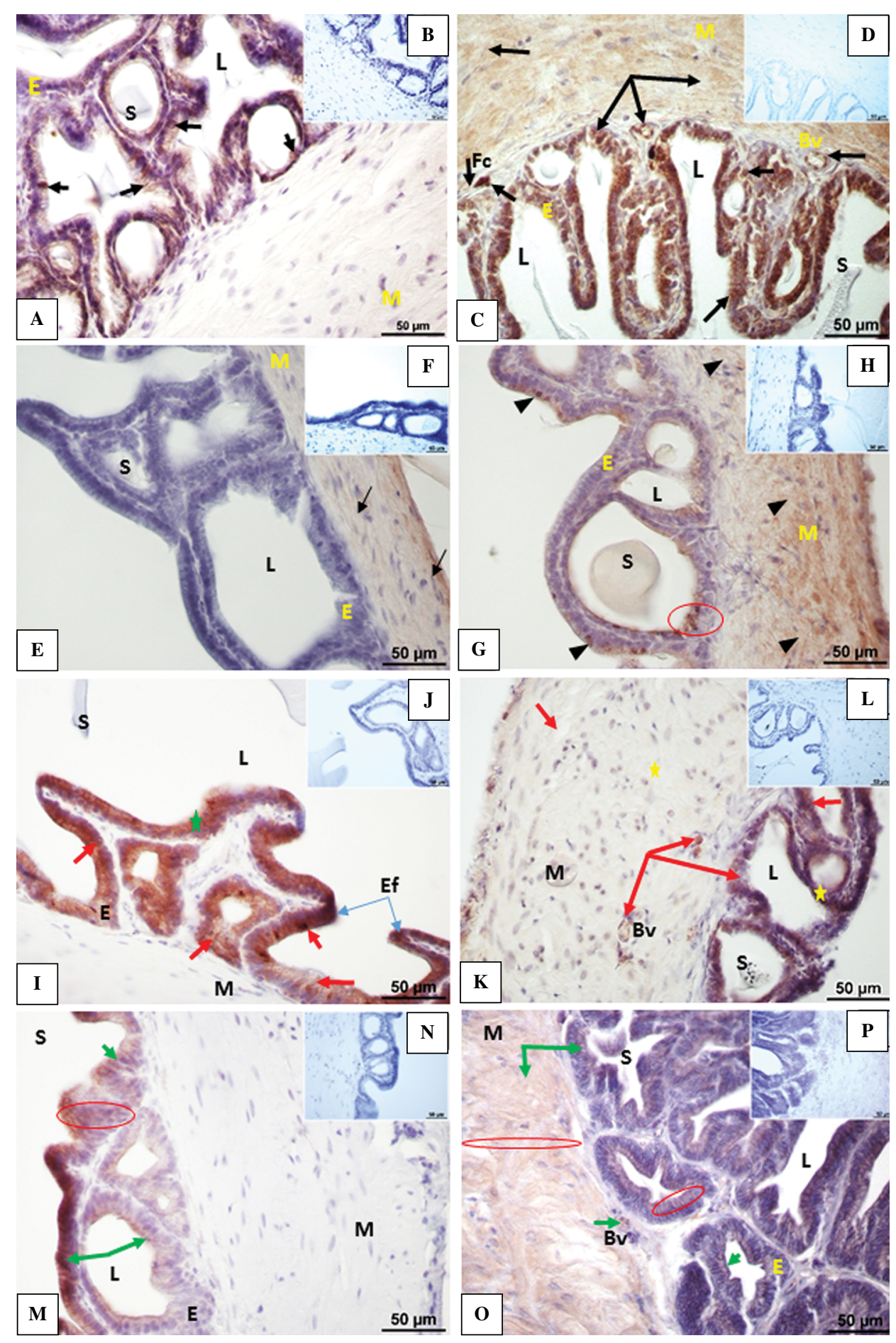

Figure 5. Immunohistochemical expression of metalloproteinases in seminal vesicles of the control and methionine-treated rats. A. Control rat: moderate MMP-2 expression in epithelial cells (nuclear and cytoplasmic) (black arrow); C. Methionine-treated rat with strong MMP-2 expression in epithelial cells and SMC and weak staining in BV (black arrow); E. Control rat with a weak MMP-9 immunoreactivity in SMC (arrow); G. Methionine-treated rat with moderate immunoexpression of MMP-9 in epithelial cells (nuclear and cytoplasmic) and strong expression in SMC (arrowhead, red circle); I. Control rat with strong MMP-3 immunoreactivity in epithelial cells (nuclear and cytoplasmic) (arrowhead, green star); K. Methionine-treated rat with a weak expression of MMP-3 in epithelial cells (nuclear and cytoplasmic) and moderate expression in SMC and blood vessels (red arrow, yellow star); M. Control rat with moderate expression of MMP-7 in epithelial cells (nuclear and cytoplasmic) (green arrow, red circle); O. Methionine-treated rat with moderate expression of MMP-7 in cytoplasm of epithelial cells and in SMC (arrow green, red circle). Inserts: B, D, F, H, J, L, N, P- negative controls. Abbreviations as in the description of Figure 3. Histological sections were stained by immunohistochemical technique as described in Material and methods, scale bars: $50 \mu \mathrm{m}$. 


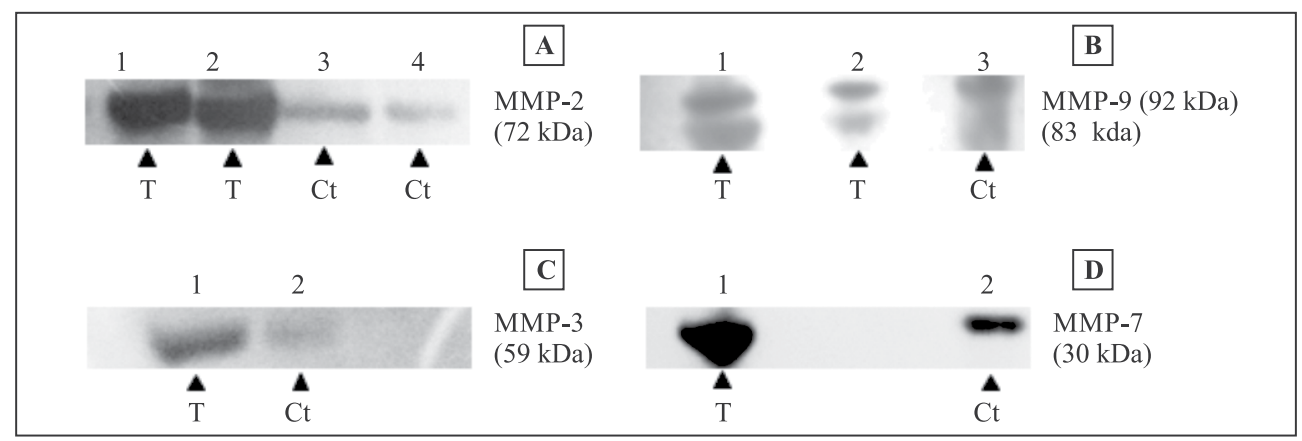

Figure 6. Representative Western blots of metalloproteinases in seminal vesicles of the control and methionine-treated rats. MMP-2, MMP-3, MMP-7 and MMP-9 Western blots. A. Representative Western blot shows 72 kDa (MMP-2) bands in control rat (lanes 3 and $4, \mathrm{Ct}$ ) and high intensity bands $(72 \mathrm{kDa}$ ) in methionine-treated rat (lanes 1 and 2, T); B. Western blot shows $92 \mathrm{kDa}$ (latent MMP-9) and $83 \mathrm{kDa}$ (active MMP-9) in methionine-treated rat (lanes 1 and 2, T), whereas in control rat only $83 \mathrm{kDa}$ form was present (lane 3, Ct); C. Western blot of MMP-3, with thick intensity band expression in methionine-treated rat (Lane 1, T) as compared to the control rat (Lane 2, Ct); D. MMP-7 had high intensity band in methionine-treated rat (Fig. 6, lane 1, T) and low intensity band in control rat (Lane 2, Ct). Abbreviations: $\mathrm{T}-$ methionine-treated; $\mathrm{Ct}-\mathrm{control}$.

of epithelial cells. The methionine-treated rats revealed MMP-7-Ir in the cytoplasm of epithelial cells but never in their nuclei. In addition, MMP-7-Ir was observed in smooth muscle. Table 1 illustrates a summary of the results concerning the immunoreactivity of various analyzed MMPs in the seminal vesicles of the treated and control rats.

\section{Relative content of metalloproteinases (-2, -3, -7, -9) in the wall of seminal vesicles of control and methionine-treated rats}

The results of immunohistochemical staining showed that MMP-2, MMP-3, MMP-7 and MMP-9 expression was increased in seminal vesicles of the methionine-treated rats compared to controls (Fig. 5). To confirm these results, a Western blot analysis was conducted. MMP-2 (72 kDa form) was identified in seminal vesicles of both the control and treated rats (Fig. 6A). The highest expression was observed in the methionine-treated rats (Fig. 6A) and the lowest expression in the control rats (Fig. 6A). In the control rats, only the $92 \mathrm{kDa}$ form (latent form) was expressed in seminal vesicles (Fig. 6B). In the treated rats, both latent $(92 \mathrm{kDa})$ and active $(83 \mathrm{kDa})$ forms of MMP-9 were detected (Fig. 6B), with a higher intensity of the corresponding bands compared to the control rats. The MMP-3 protein was expressed by Western blots in the seminal vesicles of the treated and control rats. The methionine-treatment induced an elevation of MMP-3 expression in the treated rats compared to the controls (Fig. 6C). The immunoblot depicted in Figure 6D reveals the presence of a high intensity band corresponding to MMP-7 in the treated rats compared to the control animals.
It should be noticed that in all cases, methionine induced an elevation of MMP expression, as thicker bands are observed in the treated rats compared to the control rats (Fig. 6A-D).

\section{Discussion}

Elevated plasma homocysteine concentration is considered a risk factor for cardiovascular disease, renal failure and other diseases [31, 32]. Moreover, plasma Hcy level positively correlated with remodeling of extracellular matrix (ECM) of several tissues, including heart and liver [33, 34]. According to our knowledge, no study investigated the effect of Hhcy on seminal vesicles morphology. In the present study, we investigated the expression of MMPs $(-2,-3,-7$ and -9$)$ and collagens (I and III) in the seminal vesicles of rats submitted for 6 months to a methionine-enriched diet in order to evaluate the effects of hyperhomocysteinemia on ECM remodeling. The histochemical study of the seminal vesicles revealed accumulation of collagen fibers that was associated with the deposition of glycoproteins in connective tissue and few changes of proteoglycans pattern in the treated rats. In addition, the immunohistochemical study showed the accumulation of type I and III collagens and increased expression of MMP-2, -3, -7, -9 in methionine-treated rats. The Western blot analysis also showed an activation of MMP-9 (active forms).

It was demonstrated that high methionine intake led to significant increase of Hcy in plasma, showing that methionine is a crucial factor for the installation of Hhcy [35]. In humans, a single orally high administration of methionine $(0.1 \mathrm{~g} / \mathrm{kg})$ resulted in a mild 
Hhcy condition [36]. In animals, a high methionine diet supplemented for 6 months caused Hhcy [37]. Our finding of a 2-fold elevation of total plasma Hcy level corresponds with the results of previous studies on experimental hyperhomocysteinemia model, which reported increased plasma homocysteine levels in rats on the methionine-supplemented diets $[38,39]$.

Our study demonstrated that elevated homocysteine levels were associated with a deposition of ECM proteins in seminal vesicles. Both the histochemical and immunohistochemical investigations revealed increased expression of collagen types I and III in the methionine-treated rats compared to the controls. Thus, these results suggest that increased Hcy synthesis following methionine administration mediates development of fibrosis in seminal vesicles of Wistar rats. Fibrosis is a result of an imbalance in a dynamic process of both matrix deposition and degradation. Several authors [40-42] reported that Hcy increased the collagen synthesis and its accumulation in the cardiac tissue, kidney and carotid vein, in hyperhomocysteinemic animals compared with control. An in vitro study confirmed these results in aortic smooth muscle cells from a young male rabbit [43]. However, the mechanisms by which Hcy upregulates the synthesis of collagen and its accumulation in tissue have not been still completely identified. Several authors suggested that increased oxidative stress due to Hcy could induce an imbalance between metalloproteinases and tissue inhibitors of metalloproteinases (TIMPS). These molecules are known to control the collagen turnover and its concentration in tissues [44, 45]. These MMPs are involved in the ECM remodeling, which is characteristic of many physiological and pathological processes including, development, morphogenesis, placental development, cancer progression, spermatogenesis and maturation of sperm [46-48]. Furthermore, it has been shown that Hcy modulates the synthesis, secretion, and activity of MMPs in several tissues and cells [49-51]. In this regard, a relationship between Hhcy and MMPs in seminal vesicles could be suggested by the present study since hyperhomocysteinemic rats showed increased expression of MMP-2, MMP-3, MMP-7 and MMP-9 in seminal vesicles. Traditionally, the MMPs have been considered mainly as proteases involved in the degradation of ECM proteins; however, some MMPs are indeed pro-fibrotic (stimulatory), whereas others can have anti-fibrotic (inhibitory) functions [51]. For example, gelatinase (A and B), matrilysin and stromelysin-1 are overexpressed in several diseases, including idiopathic pulmonary fibrosis and hepatic fibrosis [52-55]. They have a pro-fibrotic effect on cytokine release, including transforming growth factor $\beta$
(TGF $\beta$ ), which leads to an increased production and/or reduced degradation of ECM [26]. Based on the results of this study, it can be suggested that MMPs activation in the seminal vesicles has a profibrotic role, leading to fibrosis. Numerous studies reported that Hhcy induced the expression of matrix MMPs in several cell types, including cardiomyocytes, macrophages, smooth muscle cells and endothelial cells $[56,57]$. Moreover, other researches found that the activation of MMPs in Hhcy exerted a strong influence on ECM remodeling in vascular diseases [58]. For example, Hhcy-mediated impairment of aortic tissue from Wistar rat by the alteration of elastin/collagen ratio and the activation of MMP-2 [59]. Another study also has demonstrated that Hhcy could increase the atherosclerotic lesions by inducing fibrosis and increasing the activity of MMP-9 in murine aorta [60]. These results support the hypothesis that the exposure to elevated levels of Hcy promotes ECM remodeling through the increased MMPs activation.

\section{Conclusions}

In rats under long-term methionine-enriched diet the increased plasma homocysteine levels are associated with increased expression of metalloproteinases and collagen I and III deposition within the lamina propria and surrounding smooth muscle cells leading to the development of fibrosis in seminal vesicles. Thus, hyperhomocysteinemia could negatively affect male reproductive system through the disruption of ECM homeostasis.

\section{Conflicts of interest}

The authors indicate no potential conflict of interests.

\section{Acknowledgements}

The authors also acknowledge the technical assistance provided by Leila Khedis and Caroline Bouchot.

\section{References}

1. Finkelstein JD. Pathways and regulation of homocysteine metabolism in mammals. Semin Thromb Hemost. 2000; 26(3): 219-225, doi: 10.1055/s-2000-8466, indexed in Pubmed: 11011839 .

2. Boushey CJ, Beresford SA, Omenn GS, et al. A quantitative assessment of plasma homocysteine as a risk factor for vascular disease. Probable benefits of increasing folic acid intakes. JAMA. 1995; 274(13): 1049-1057, doi: 10.1001/ /jama.274.13.1049, indexed in Pubmed: 7563456.

3. Bukharaeva E, Shakirzyanova A, Khuzakhmetova V, et al. Homocysteine aggravates ROS-induced depression of transmitter release from motor nerve terminals: potential mechanism of peripheral impairment in motor neuron diseases 
associated with hyperhomocysteinemia. Front Cell Neurosci. 2015; 9: 391, doi: 10.3389/fncel.2015.00391, indexed in Pubmed: 26500495

4. Xie Di, Yuan Y, Guo J, et al. Hyperhomocysteinemia predicts renal function decline: a prospective study in hypertensive adults. Sci Rep. 2015; 5: 16268, doi: 10.1038/srep16268, indexed in Pubmed: 26553372

5. Mendes RH, Mostarda C, Candido GO, et al. Moderate hyperhomocysteinemia provokes dysfunction of cardiovascular autonomic system and liver oxidative stress in rats. Auton Neurosci. 2014; 180: 43-47, doi: 10.1016/j.autneu.2013.10.006, indexed in Pubmed: 24231341.

6. Li JJ, Li Q, Du HP, et al. Homocysteine triggers inflammatory responses in macrophages through inhibiting CSE-H2S signaling via DNA hypermethylation of CSE promoter. Int J Mol Sci. 2015; 16(6): 12560-12577, doi: 10.3390/ijms160612560, indexed in Pubmed: 26047341

7. Matté C, Stefanello FM, Mackedanz V, et al. Homocysteine induces oxidative stress, inflammatory infiltration, fibrosis and reduces glycogen/glycoprotein content in liver of rats. Int J Dev Neurosci. 2009; 27(4): 337-344, doi: 10.1016/j. ijdevneu.2009.03.005, indexed in Pubmed: 19460627.

8. Pushpakumar S, Kundu S, Narayanan N, et al. DNA hypermethylation in hyperhomocysteinemia contributes to abnormal extracellular matrix metabolism in the kidney. FASEB J. 2015; 29(11): 4713-4725, doi: 10.1096/fj.15-272443, indexed in Pubmed: 26224753.

9. Nagase H, Woessner JF. Matrix metalloproteinases. J Biol Chem. 1999; 274(31): 21491-21494, doi: 10.1074/jbc.274.31. 21491.

10. Chakrabarti S, Patel KD. Matrix metalloproteinase-2 (MMP2) and MMP-9 in pulmonary pathology. Exp Lung Res. 2005; 31(6): 599-621, doi: 10.1080/019021490944232, indexed in Pubmed: 16019990.

11. Collier IE, Wilhelm SM, Eisen AZ, et al. H-ras Oncogene-transformed human bronchial epithelial cells (TBE-1) secrete a single metalloprotease capable of degrading basement membrane collagen. J Biol Chem. 1988; 263: 5579-6587, indexed in Pubmed: 2834383.

12. Lewkowicz J, Knapp M, Tankiewicz-Kwedlo A, et al. MMP-9 in atrial remodeling in patients with atrial fibrillation. Ann Cardiol Angeiol (Paris). 2015; 64(4): 285-291, doi: 10.1016/j. ancard.2014.12.004, indexed in Pubmed: 25869465.

13. Giannandrea M, Parks WC. Diverse functions of matrix metalloproteinases during fibrosis. Dis Model Mech. 2014; 7(2): 193-203, doi: 10.1242/dmm.012062, indexed in Pubmed: 24713275 .

14. Johansson N, Saarialho-Kere U, Airola K, et al. Collagenase-3 (MMP-13) is expressed by hypertrophic chondrocytes, periosteal cells, and osteoblasts during human fetal bone development. Develop Dyn. 1997; 208(3): 387-397, doi: 10.1002/ (sici)1097-0177(199703)208:3<387::aid-aja9>3.0.co;2-e.

15. Li Jr, Shen T, Wang Yl, et al. Expression of matrix metalloproteinases and ovarian morphological changes in androgenized cyclic female guinea pigs. Tissue Cell. 2016; 48(1): 72-80, doi: 10.1016/j.tice.2015.10.003, indexed in Pubmed: 26710991.

16. Farina AR, Cappabianca L, DeSantis G, et al. Thioredoxin stimulates MMP-9 expression, de-regulates the MMP-9/ /TIMP-1 equilibrium and promotes MMP-9 dependent invasion in human MDA-MB-231 breast cancer cells. FEBS Lett. 2011; 585(20): 3328-3336, doi: 10.1016/j.febslet.2011.09.023, indexed in Pubmed: 21963718.

17. Chang JH, Huang $\mathrm{YH}$, Cunningham $\mathrm{CM}$, et al. Matrix metalloproteinase 14 modulates signal transduction and angiogenesis in the cornea. Surv Ophthalmol. 2016; 61(4):
478-497, doi: 10.1016/j.survophthal.2015.11.006, indexed in Pubmed: 26647161.

18. Nguyen AH, Wang Y, White DE, et al. MMP-mediated mesenchymal morphogenesis of pluripotent stem cell aggregates stimulated by gelatin methacrylate microparticle incorporation. Biomaterials. 2016; 76: 66-75, doi: 10.1016/j.biomaterials.2015.10.043, indexed in Pubmed: 26519649.

19. Gaide Chevronnay HP, Selvais C, Emonard H, et al. Regulation of matrix metalloproteinases activity studied in human endometrium as a paradigm of cyclic tissue breakdown and regeneration. Biochim Biophys Acta. 2012; 1824(1): 146-156, doi: 10.1016/j.bbapap.2011.09.003, indexed in Pubmed: 21982799.

20. Zhang X, Wang HM, Lin HY, et al. Regulation of matrix metalloproteinases (MMPS) and their inhibitors (TIMPS) during mouse peri-implantation: role of nitric oxide. Placenta. 2004; 25(4): 243-252, doi: 10.1016/j.placenta.2003.08.014, indexed in Pubmed: 15028415.

21. Balbín M, Fueyo A, Knäuper V, et al. Analysis of its potential role in postpartum involution of the uterus. J Biol Chem. 1998; 273: 23959-23968, doi: 10.1016/j.placenta.2003.08.014.

22. Ferrer M, Rodriguez H, Zara L, et al. MMP2 and acrosin are major proteinases associated with the inner acrosomal membrane and may cooperate in sperm penetration of the zona pellucida during fertilization. Cell Tissue Res. 2012; 349(3): 881-895, doi: 10.1007/s00441-012-1429-1, indexed in Pubmed: 22729485.

23. Slongo ML, Zampieri M, Onisto M. Expression of matrix metalloproteases (MMP-2, MT1 -MMP) and their tissue inhibitor (TIMP-2) by rat sertoli cells in culture: implications for spermatogenesis. Biol Chem. 2002; 383(1): 235-239, doi: 10.1515/ BC.2002.025, indexed in Pubmed: 11928819.

24. Tentes I, Asimakopoulos B, Mourvati E, et al. Matrix metalloproteinase (MMP)-2 and MMP-9 in seminal plasma. J Assist Reprod Genet. 2007; 24(7): 278-281, doi: 10.1007/ s10815-007-9129-6, indexed in Pubmed: 17616803.

25. Belhocine M, Gernigon-Spychalowicz T, Robert AM, et al. Ecophysiological responses of the seminal vesicle of Libyan jird (Meriones libycus) to the Saharan conditions: histologi$\mathrm{cal}$, morphometric and immunohistochemical analysis. Histol Histopathol. 2007; 22(6): 603-615, doi: 10.14670/HH-22.603, indexed in Pubmed: 17357090.

26. Raaf L, Noll C, Cherifi ME, et al. Myocardial fibrosis and TGFB expression in hyperhomocysteinemic rats. Mol Cell Biochem. 2011; 347(1-2): 63-70, doi: 10.1007/s11010-0100612-5, indexed in Pubmed: 20938722.

27. Shipchandler MT, Moore EG. Rapid, fully automated measurement of plasma homocyst(e)ine with the Abbott IMx analyzer. Clin Chem. 1995; 41(7): 991-994, indexed in Pubmed: 7600701.

28. Junqueira LC, Bignolas G, Brentani RR. Picrosirius staining plus polarization microscopy, a specific method for collagen detection in tissue sections. Histochem J. 1979; 11(4): 447-455, doi: 10.1007/BF01002772, indexed in Pubmed: 91593.

29. Mowry RW. Alcian blue techniques for the histochemical study of acidic carbohydrates. J Histochem Cytochem. 1956; 4: 407-408, indexed in Pubmed: 10011553567.

30. Gabe M. Techniques histologiques: chapitre 15, Detection histochimiques des aldéhydes et cétones. Ed Masson et Cie, Paris 1968: p368.

31. Suszyńska-Zajczyk J, Utyro O, Jakubowski H. Methionine-induced hyperhomocysteinemia and bleomycin hydrolase deficiency alter the expression of mouse kidney proteins involved in renal disease. Mol Genet Metab. 2014; 112(4): 339-346, doi: 10.1016/j.ymgme.2014.05.010, indexed in Pubmed: 24913063. 
32. Basu A, Jenkins AJ, Stoner JA, et al. DCCT/EDIC Research Group. Plasma total homocysteine and carotid intima-media thickness in type 1 diabetes: a prospective study. Atherosclerosis. 2014; 236(1): 188-195, doi: 10.1016/j.atherosclerosis.2014.07.001, indexed in Pubmed: 25063949.

33. Joseph J, Washington A, Joseph L, et al. Hyperhomocysteinemia leads to adverse cardiac remodeling in hypertensive rats. Am J Physiol Heart Circ Physiol. 2002; 283(6): H2567-H2574, doi: 10.1152/ajpheart.00475.2002, indexed in Pubmed: 12388235.

34. Matté C, Stefanello FM, Mackedanz V, et al. Homocysteine induces oxidative stress, inflammatory infiltration, fibrosis and reduces glycogen/glycoprotein content in liver of rats. Int J Dev Neurosci. 2009; 27(4): 337-344, doi: 10.1016/j. ijdevneu.2009.03.005, indexed in Pubmed: 19460627.

35. Fukada Si, Shimada Y, Morita T, et al. Suppression of methionine-induced hyperhomocysteinemia by glycine and serine in rats. Biosci Biotechnol Biochem. 2006; 70(10): 2403-2409, doi: $10.1271 / \mathrm{bbb} .60130$, indexed in Pubmed: 17031061.

36. Bellamy MF, McDowell IF, Ramsey MW, et al. Hyperhomocysteinemia after an oral methionine load acutely impairs endothelial function in healthy adults. Circulation. 1998; 98(18): 1848-1852, doi: 10.1161/01.CIR.98.18.1848, indexed in Pubmed: 9799203.

37. Yalçinkaya S, Unlüçerçi $Y$, Giriş $M$, et al. Oxidative and nitrosative stress and apoptosis in the liver of rats fed on high methionine diet: protective effect of taurine. Nutrition. 2009; 25(4): 436-444, doi: 10.1016/j.nut.2008.09.017, indexed in Pubmed: 19056240.

38. Sharma M, Rai SK, Tiwari M, et al. Effect of hyperhomocysteinemia on cardiovascular risk factors and initiation of atherosclerosis in Wistar rats. Eur J Pharmacol. 2007; 574(1): 49-60, doi: 10.1016/j.ejphar.2007.07.022, indexed in Pubmed: 17706635.

39. Xu Y, Tian Ye, Wei HJ, et al. Methionine diet-induced hyperhomocysteinemia accelerates cerebral aneurysm formation in rats. Neurosci Lett. 2011; 494(2): 139-144, doi: 10.1016/j. neulet.2011.02.076, indexed in Pubmed: 21382440.

40. Joseph J, Kennedy RH, Devi S, et al. Protective role of mast cells in homocysteine-induced cardiac remodeling. Am J Physiol Heart Circ Physiol. 2005; 288(5): H2541-H2545, doi: 10.1152/ /ajpheart.00806.2004, indexed in Pubmed: 15591099.

41. Cao Lu, Lou X, Zou Z, et al. Folic acid attenuates hyperhomocysteinemia-induced glomerular damage in rats. Microvasc Res. 2013; 89: 146-152, doi: 10.1016/j.mvr.2013.07.002, indexed in Pubmed: 23859838.

42. Tan H, Shi C, Jiang X, et al. Hyperhomocysteinemia promotes vascular remodeling in vein graph in mice. Front Biosci (Landmark Ed). 2014; 19: 958-966, indexed in Pubmed: 24896329.

43. Tyagi N, Sedoris KC, Steed M, et al. Mechanisms of homocysteine-induced oxidative stress. Am J Physiol Heart Circ Physiol. 2005; 289(6): H2649-H2656, doi: 10.1152/ ajpheart.00548.2005, indexed in Pubmed: 16085680.

44. Kundu S, Tyagi N, Sen U, et al. Matrix imbalance by inducing expression of metalloproteinase and oxidative stress in cochlea of hyperhomocysteinemic mice. Mol Cell Biochem. 2009; 332(1-2): 215-224, doi: 10.1007/s11010-009-0194-2, indexed in Pubmed: 19590937.

45. Nuttall RK, Sampieri CL, Pennington CJ, et al. Expression analysis of the entire MMP and TIMP gene families during mouse tissue development. FEBS Lett. 2004; 563(1-3): 129-134, doi: 10.1016/S0014-5793(04)00281-9, indexed in Pubmed: 15063736.

46. Nguyen AH, Wang Y, White DE, et al. MMP-mediated mesenchymal morphogenesis of pluripotent stem cell aggregates stimulated by gelatin methacrylate microparticle incorporation. Biomaterials. 2016; 76: 66-75, doi: 10.1016/j.biomaterials.2015.10.043, indexed in Pubmed: 26519649.
47. Walter I, Schönkypl S. Extracellular matrix components and matrix degrading enzymes in the feline placenta during gestation. Placenta. 2006; 27(2-3): 291-306, doi: 10.1016/j.placenta.2005.02.014, indexed in Pubmed: 16338474.

48. Grünwald B, Vandooren J, Locatelli E, et al. Matrix metalloproteinase-9 (MMP-9) as an activator of nanosystems for targeted drug delivery in pancreatic cancer. J Control Release. 2016; 239: 39-48, doi: 10.1016/j.jconrel.2016.08.016, indexed in Pubmed: 27545397.

49. Kim SH, Song YS, Hwang SY, et al. Effects of hormones on the expression of matrix metalloproteinases and their inhibitors in bovine spermatozoa. Asian-Australas J Anim Sci. 2013; 26(3): 334-342, doi: 10.5713/ajas.2012.12564, indexed in Pubmed: 25049795.

50. Guo H, Lee JD, Uzui H, et al. Effects of heparin on the production of homocysteine-induced extracellular matrix metalloproteinase- 2 in cultured rat vascular smooth muscle cells. Can J Cardiol. 2007; 23(4): 275-280, indexed in Pubmed: 17380220 .

51. Tyagi N, Kandel M, Munjal C, et al. Homocysteine mediated decrease in bone blood flow and remodeling: role of folic acid. J Orthop Res. 2011; 29(10): 1511-1516, doi: 10.1002/ /jor.21415, indexed in Pubmed: 21469179.

52. Chaussalet M, Lamy E, Foucault-Bertaud A, et al. Homocysteine modulates the proteolytic potential of human vascular endothelial cells. Biochem Biophys Res Commun. 2004; 316(1): 170-176, doi: 10.1016/j.bbrc.2004.02.027, indexed in Pubmed: 15003526.

53. Torres L, García-Trevijano ER, Rodríguez JA, et al. Induction of TIMP-1 expression in rat hepatic stellate cells and hepatocytes: a new role for homocysteine in liver fibrosis. Biochim Biophys Acta. 1999; 1455(1): 12-22, indexed in Pubmed: 10524225 .

54. Wang ZS, Jin H, Wang DM. Influence of hydrogen sulfide on zymogen activation of homocysteine-induced matrix metalloproteinase-2 in H9C2 cardiocytes. Asian Pac J Trop Med. 2016; 9(5): 489-493, doi: 10.1016/j.apjtm.2016.03.023, indexed in Pubmed: 27261860.

55. Lee SJ, Lee YiS, Seo KW, et al. Homocysteine enhances MMP-9 production in murine macrophages via ERK and Akt signaling pathways. Toxicol Appl Pharmacol. 2012; 260(1): 89-94, doi: 10.1016/j.taap.2012.01.026, indexed in Pubmed: 22326992.

56. Doronzo G, Russo I, Mattiello L, et al. Homocysteine rapidly increases matrix metalloproteinase-2 expression and activity in cultured human vascular smooth muscle cells. Role of phosphatidyl inositol 3-kinase and mitogen activated protein kinase pathways. Thromb Haemost. 2005; 94(6): 1285-1293, doi: 10.1160/TH05-04-0221, indexed in Pubmed: 16411407.

57. Tyagi SC, Meyer L, Schmaltz RA, et al. Proteinases and restenosis in the human coronary artery: extracellular matrix production exceeds the expression of proteolytic activity. Atherosclerosis. 1995; 116(1): 43-57, indexed in Pubmed: 7488332.

58. Ke XD, Foucault-Bertaud A, Genovesio C, et al. Homocysteine modulates the proteolytic potential of human arterial smooth muscle cells through a reactive oxygen species dependant mechanism. Mol Cell Biochem. 2010; 335(1-2): 203-210, doi: 10.1007/s11010-009-0270-7, indexed in Pubmed: 19787299

59. Mujumdar VS, Aru GM, Tyagi SC. Induction of oxidative stress by homocyst(e)ine impairs endothelial function. J Cell Biochem. 2001; 82(3): 491-500, indexed in Pubmed: 11500925.

60. Hofmann MA, Lalla E, Lu Y, et al. Hyperhomocysteinemia enhances vascular inflammation and accelerates atherosclerosis in a murine model. J Clin Invest. 2001; 107(6): 675-683, doi: 10.1172/JCI10588, indexed in Pubmed: 11254667.

Submitted: 12 May, 2016

Accepted after reviews: 13 June, 2017 Available as AoP: 20 June, 2017 